\title{
Erratum to: Trends in the Mechanistic and Dynamic Modeling of Infectious Diseases
}

\author{
Justin Lessler ${ }^{1}$ • Andrew S. Azman ${ }^{1}$ - M. Kate Grabowski ${ }^{1}$ • \\ Henrik Salje $^{1}$ - Isabel Rodriguez-Barraquer ${ }^{1}$
}

Published online: 5 August 2016

(C) Springer International Publishing AG 2016

Erratum to: Curr Epidemiol Rep (2016)

DOI: 10.1007/s40471-016-0078-4

Due to an oversight in proofing, symbols in Box 1 in the original article did not render correctly inline to the text. Please see the corrected Box 1 listed here.

The online version of the original article can be found at 10.1007/s40471016-0078-4.

$\triangle$ Justin Lessler justin@jhu.edu

1 Johns Hopkins Bloomberg School of Public Health, Baltimore,

MD, USA 


\section{Drivers of Infectious Disease Dynamics}

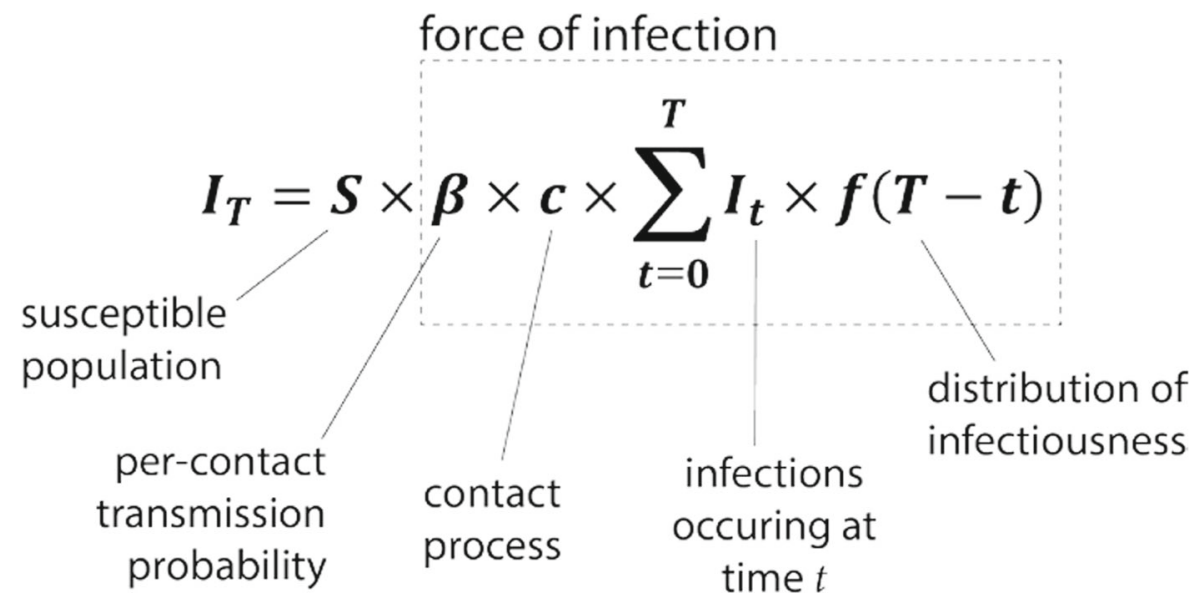

The epidemic dynamics of infectious diseases are driven by similar mechanistic relationships between the current health state of the population. The expected number of infections at some time $T$ is illustrated for a directly transmitted disease in the above equation. Dynamic and mechanistic models of disease spread, regardless of complexity, capture these relationships in order to improve inference or predict the disease dynamics. The study of infectious disease dynamics encompasses the study of any of the shared drivers of the mechanistic processes of disease spread with an eye towards better understanding disease transmission. As illustrated above, these include:

The size of the susceptible population $(\boldsymbol{S})$ : The number of people available to be infected. The dynamics of susceptibility is not shown here, but can itself can be complex, as new susceptibles enter the population through birth, immigration and loss of immunity. For many diseases (e.g., dengue, influenza), susceptibility is not a binary state, and complex models may be needed.

The force of infection: The force of infection is the probability that any individual who is susceptible at a given time becomes infected (analogous to the hazard of infection). The size of the susceptible population times the force of infection is the reproductive number $(R)$. When this value is above 1 , the epidemic will grow. When it falls below 1 , it will recede.

The infectious process $(\boldsymbol{\beta})$ : The infectious process dictates the chances of becoming infected on a direct or indirect contact with an infectious individual. Here represented as a per contact probability of infection, this itself can be a complex, multi-faceted process.

The contact process $(\boldsymbol{c})$ : The process by which infectious contacts are made, whether directly or mediated by a some vector or the environment, is one of the most complex parts of the infectious process. Much modern research focuses on accounting the role of space and population structure in the contact process.

Previous infections $\left(I_{t}\right)$ : Fundamental to the nature of infectious diseases is the number of previous infections, however, these may not as directly lead to current infections as illustrated here if transmission is mediated by a vector or the environment.

The natural history of disease $(\boldsymbol{f}(\boldsymbol{T}-\boldsymbol{t}))$ : How infectious people are at particular times after their infection determines their contribution to ongoing disease transmission, and fundamentally drives the speed at which epidemics move through the population. Other aspects of disease natural history (e.g., the incubation period) may determine our ability to control a disease and its ultimate health impact. 